\title{
The big and the small picture
}

DOI:

$10.1038 /$ nrc2176

URLs
ATM
http://ca.expasy.org/uniprot/
Q13315

ATR

http://ca.expasy.org/uniprot/ Q13535

BRCA1

http://ca.expasy.org/uniprot/

P38398

53BP1

http://www.ncbi.nlm.nih.gov/ sites/entrez?Db=gene\&Cmd=S howDetailViewETermToSearch $=7158$ 8itool=EntrezSystem 2 .

PEntrez.Gene.Gene

ResultsPanel.Gene_RVDocSum

TOPBP1

http://www.ncbi.nlm.nih.gov/ sites/entrez?Db=gene\&Cmd=S howDetailView\&TermToSearch $=11073$ \&itool=EntrezSystem 2 . PEntrez.Gene.Gene

ResultsPanel.Gene_RVDocSum

RAP80

http://ca.expasy.org/uniprot/

Q96RL1

\section{BACH1}

http://www.ncbi.nlm.nih.gov/ sites/entrez?Db=gene\&Cmd=S howDetailViewGTermToSearch $=571$ Eitool $=$ EntrezSystem 2 .

PEntrez.Gene.Gene

ResultsPanel.Gene_RVDocSum

CTIP

http://www.ncbi.nlm.nih.gov/ sites/entrez?Db=gene\& $\mathrm{Cmd}=\$$ howDetailView\&TermToSearch $=$ 5932\&itool=EntrezSystem 2 . PEntrez.Gene.Gene

ResultsPanel.Gene_RVDocSum
Systems biology approaches can reveal the broad range of players that are involved in a cellular process and, at the same time, open avenues of investigation around specific players, as is nicely illustrated by four reports in Science.

Matsuoka et al. carried out a largescale proteomics analysis of proteins that were phosphorylated in response to DNA damage on consensus sites recognized by ATM and ATR kinases. They identified $>900$ DNA-damage-inducible phosphorylation sites on $>700$ proteins. The extent of phosphorylation of known ATM and ATR substrates was much higher than anticipated; for example, among the mediator class of proteins (including BRCA1, 53BP1 and TOPBP1), 33 regulated sites were found, of which only six had been previously identified.

The phosphorylation of a small subset of candidate substrates was validated by immunoprecipitation and western blot. In addition, the authors chose a random selection of substrates that had not previously been implicated in the DNA damage response and tested cells that were depleted for these substrates in functional assays. Out of 37 proteins, 35 scored in at least one of four assays and more than half of the proteins scored in two or more assays - suggesting that the pool of candidate substrates is enriched with proteins that function in the DNA damage response.

Using gene-ontology analysis, 421 out of $>700$ candidate substrates were annotated with a biological process. Among these, 202 substrates were assigned to the nucleic-acid metabolism category, of which 46 have a function in DNA replication, repair or recombination and 101 have a function in mRNA transcription. The post-transcriptional modification category was surprisingly large. Together, these analyses imply the existence of a broad transcriptional, post-transcriptional and chromatin response to DNA damage. Many of the substrates cluster into protein

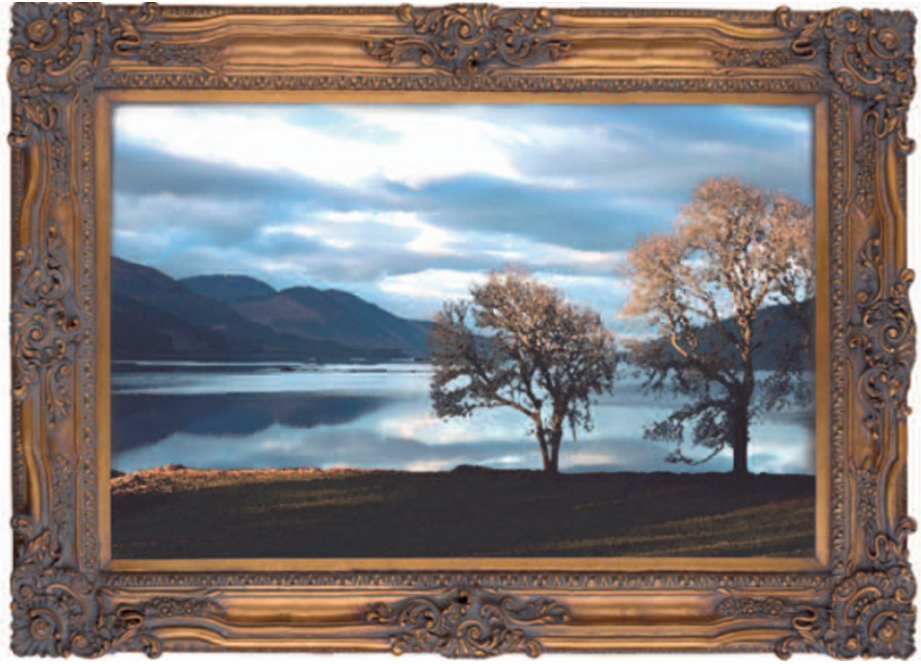

networks that are involved in DNA replication, DNA repair, the cell cycle and the spindle checkpoint. Several signalling pathways were also implicated in the DNA damage response. Elucidation of these many newly found connections will take time, but this study demonstrates the extraordinarily broad cellular response to DNA damage.

This DNA-damage phosphorylation information has already proved useful in identifying two ATM/ATR substrates as binding partners of the tumour suppressor BRCA1. The BRCT repeats of BRCA1 constitute a phosphopeptide recognition domain, which was used by Wang et al. to affinity-purify phosphopeptides and identify the novel protein Abraxas and the ubiquitin-interacting motif (UIM)-containing protein RAP80. It is thought that RAP80 is recruited to BRCA1 through binding to Abraxas. Functional analyses showed that both proteins are required for DNA damage resistance, control of the G2-M checkpoint and DNA repair.

Two other groups, led by Bijan Sobhian and Hongtae Kim, respectively, also identified RAP80 as a BRCA1 interaction partner. Mutational analysis revealed that the UIM domains are required for colocalization of RAP80 to double-strand breaks (DSBs) and for polyubiquitin binding. All three groups showed that RAP80 is responsible for recruiting BRCA1 to DNA damage-induced foci. Together, these findings suggest that RAP80 targets the BRCA1 complex to polyubiquitylated structures at DSBs, although the nature of these polyubiquitylated substrates remains unclear.

Given that BRCA1 has additional interaction partners, including $\mathrm{BACH} 1$ and CTIP, it is proposed to exist in distinct, mutually exclusive complexes. It will be interesting to dissect the role of each of these complexes in the DNA damage response and in tumorigenesis.

Arianne Heinrichs, Chief Editor

Nature Reviews Molecular Cell Biology

ORIGINAL RESEARCH PAPERS Matsuoka, S. et al. ATM and ATR substrate analysis reveals extensive protein networks responsive to DNA damage. Science 316, 1160-1166 (2007) |Wang, B. et al. Abraxas and RAP 80 form a BRCA1 protein complex required for the DNA damage response. Science 316, 1194-1198 (2007) | Sobhian, B. et al. RAP80 targets BRCA1 to specific ubiquitin structures at DNA damage sites. Science $\mathbf{3 1 6}$, 1198-1202 (2007) | Kim, H. et al. Ubiquitinbinding protein RAP 80 mediates BRCA1dependent DNA damage response. Science $\mathbf{3 1 6}$ 1202-1205 (2007) 